\title{
Can soap films be used as models for mortality studies?
}

Benjamin Haffner, Jonathan Lalieu, Peter Richmond and Stefan Hutzler

School of Physics, Trinity College Dublin, The University of Dublin, Ireland.

Email: haffnerb@tcd.ie

\begin{abstract}
We report studies of the life expectancy of sets of parallel liquid films made from surfactant solution and contained in cylindrical perspex tubes. The tubes are sealed at the bottom and vertically placed. From our analysis of the life-time data of over 2200 such films we find that this synthetic system shows characteristics of the statistics of human mortality. An infant or wearin phase with decreasing mortality is succeeded by an ageing phase that broadly follows the law of Gompertz. Ensembles of soap films thus offer themselves as a unique and easily accessible experimental system for the study of failure/mortality statistics with applications to human data.

Key-words: thin liquid films; survival distribution, mortality; Gompertz, Weibull

Generally, for real systems, such large quantities of data is hard to collect. In particular, the age at death for human being is rarely given with precision.

In comparison, soap films are easy to produce and monitor, they can be used as a reproducible system for statistical studies.
\end{abstract}




\section{Introduction}

Given a population of $N$ individuals, which might be humans, animals, technical devices, or as in the case presented below, thin liquid films, ageing here concerns the decay of this population with time $t$, due to death, technical failure or film rupture. Data is often presented in the form of a survival function $s(t)$, defined by

$$
s(t)=N(t) / N(0), \quad \text { Eqn. }(1)
$$

where $N(0)$ is the number of individuals at time $t=0$. The survival function $s(t)$ thus specifies the relative number of individuals that are surviving (at least) up to time $t$. A further function that is used when describing ageing is the socalled force of mortality or death-rate, $\mu(t)$ (Gavrilov and Gavrilova, 2001). This is the (negative) relative rate of change of the survival function,

$$
\mu(t)=-1 / s(t) d s(t) / d t=-d \ln [s(t)] / d t, \quad \text { Eqn.(2) }
$$

where the minus sign ensures $\mu(t)$ remains positive $(\mu(t)$ is also called failure rate when referring to technical systems).

For humans, the force of mortality typically is of the so-called 'bath-tub' form, shown in figure 1 . The mortality rate is high at/after birth (infant mortality), but then rapidly decreases with an inverse power law until it reaches a minimum in the teenage years, see for example [Berrut et al. 2016]. Beyond this point it levels off for a short period and then begins to rise exponentially. This rise, first described by Gompertz in 1825 [Gompertz, $1825]$, has now been observed for many human populations in different time eras and regions of the world (see for example, Finch (1994), Bebbington et al. (2007)),

$$
\mu(t) \sim \exp (\alpha t), \quad \text { Eqn.(3) }
$$


where $\alpha$ is a constant.

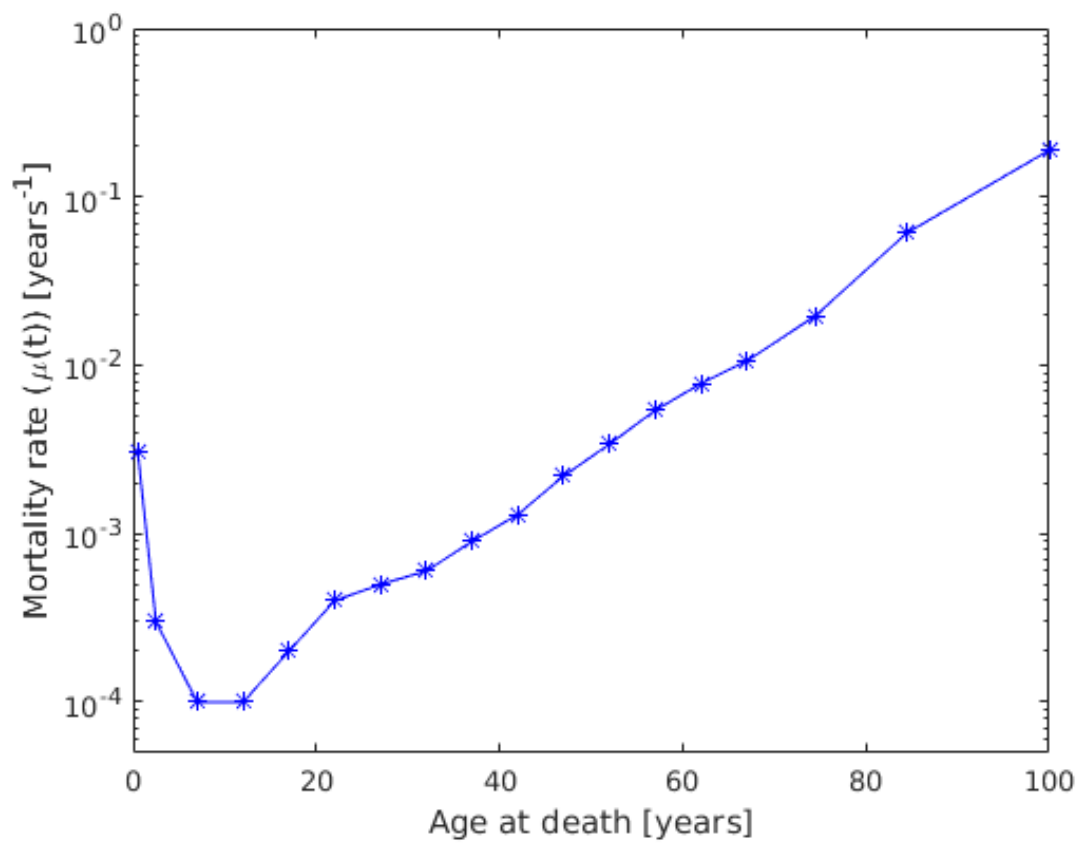

Figure 1. For humans, a (semi-log) plot of the force of mortality or death rate $\mu(t)$ (Eqn(2)) typically features a so-called 'bathtub' curve. This refers to high infant mortality, followed by a strong decrease in mortality, which begins to rise again in early teenage years (around 12 years for the data shown). In later years the rise in death-rate is approximately linear in this semi-log plot; this is referred to as the Gompertz law, Eqn(3). (The data shown here is for the French population in 2015, the raw data was taken from (INED, 2017)).

A number of models have been proposed which account for the long term Gompertz behaviour, e.g. (Gavrilov and Gavrilova, 2001, Stauffer, 2004, Shkolskii, 2005). The infant or short term behaviour is usually ascribed to defects such as congenital variations in the foetus which can result in death at birth or shortly thereafter. A key contribution of medical research has been to reduce deaths in infancy from childhood diseases which has the effect of pulling the minimum point of the bath-tub curve down to lower levels. It is more difficult to reduce the force of mortality once the death-rate begins to rise exponentially. Significant changes in this regime would seem to be likely only with radical changes in our genetic makeup. 
Some attempts to explain the shape of the bathtub curve start from so-called 'reliability theory', which enables the age-related failure kinetics of an object to be understood from analysis of its architecture and the reliability (or expected time to failure) of its component parts. If the continued viability of the object depends on the operation of each and every one of its components, such that the first failure is lethal, then the force of mortality is simply a time independent constant. This is akin to the hazard represented by extrinsic accidents, which happen without warning. If, however, the object is composed of collections of irreplaceable but redundant components, such that failure of any one does not cause death, then the object can accumulate a growing number of non-lethal component failures. Over time, this will result in an increasing probability that the last of an initially redundant cluster will fail, bringing about death. (Note that this simple model leaves out the possibility of self-repair, known from biological systems.)

Gavrilov \& Gavrilova (Gavrilov \& Gavrilova, 2016), developed this approach and showed it could predict, in the long time limit, the Gompertz form, Eqn(3). Further elaboration extended the analysis to cases where the system was at the outset compromised by a number of pre-existing faults, consistent with the fact that some inherited gene defects or mutations are transmitted to the progeny. Such an organism also follows the law of Gompertz. But where the initial number of defects or flaws was relatively low, the force of mortality in the long term rises not exponentially but according to a power law (Weibull law),

$$
\mu(t) \sim t^{\alpha} . \quad \text { Eqn.(4) }
$$

A recent review of the field has been published by Kirkwood (2014).

What is particularly interesting is that to the best of our knowledge, whilst 
mortality data is available for humans and various other biological species that support the Gompertz theory of long term mortality, Eqn(3), (with the notable exception of the non-ageing naked mole-rat (Ruby et al., 2018)), similar detailed data for mortality of synthetic systems, such as computer hard drives (Yang and Feng-Bin, 1999), or other complex systems, generally shows failure according to the Weibull law, Eqn(4). The early work of Davis (1952) discusses failure data for technical devices and errors due to mishandling of data by humans. Most of this data is described by a constant failure (mortality) rate.

The Weibull law was also used in an earlier publication of our group (Tobin et al. 2011) to describe the statistics of thin liquid films, made from surfactant solution, and confined within fully sealed cylinders. Informed by recent work on mortality (Richmond and Roehner, 2016a) we decided to reanalyse the original data, and plot it in the form of a survival function $s(t)$, together with the corresponding failure rate $\mu(t)$. To our surprise, the law of Gompertz provides a much better fit than the Weibull law, for the same number of fit parameters, namely two. Figure 2 shows the data together with fits of $s(t)$ to the two distributions on a log-lin scale. The Gompertz distribution,

$$
\begin{aligned}
& s_{G}(t)=\exp \left\{-a\left[e^{b\left(t-t_{0}\right)}-1\right]\right\}, \\
& \mu_{G}(t)=a b * e^{b\left(t-t_{0}\right)}, \quad \operatorname{Eqn}(5)
\end{aligned}
$$

provides a much better fit than the Weibull distribution,

$$
\begin{aligned}
s_{W}(t) & =\exp \left\{-\left[\left(t-t_{0}\right) / \lambda\right]^{k}\right\} \\
\mu_{W}(t)=\frac{k}{\lambda}\left(\frac{t}{\lambda}\right)^{k-1} . & \operatorname{Eqn}(6) .
\end{aligned}
$$

However, unlike in the case of human mortality, the experimental data by 
Tobin et al. (2011) does not feature a bath-tub shape, rather the Gompertz law describes the entirety of the data (i.e. $t_{0}=0$ ). Accordingly, apart from the final stages, where the lack of data introduces huge scatter when performing the numerical derivative required to compute the mortality rate, $\mu(t)$ varies linearly in the log-lin plot of figure 2.

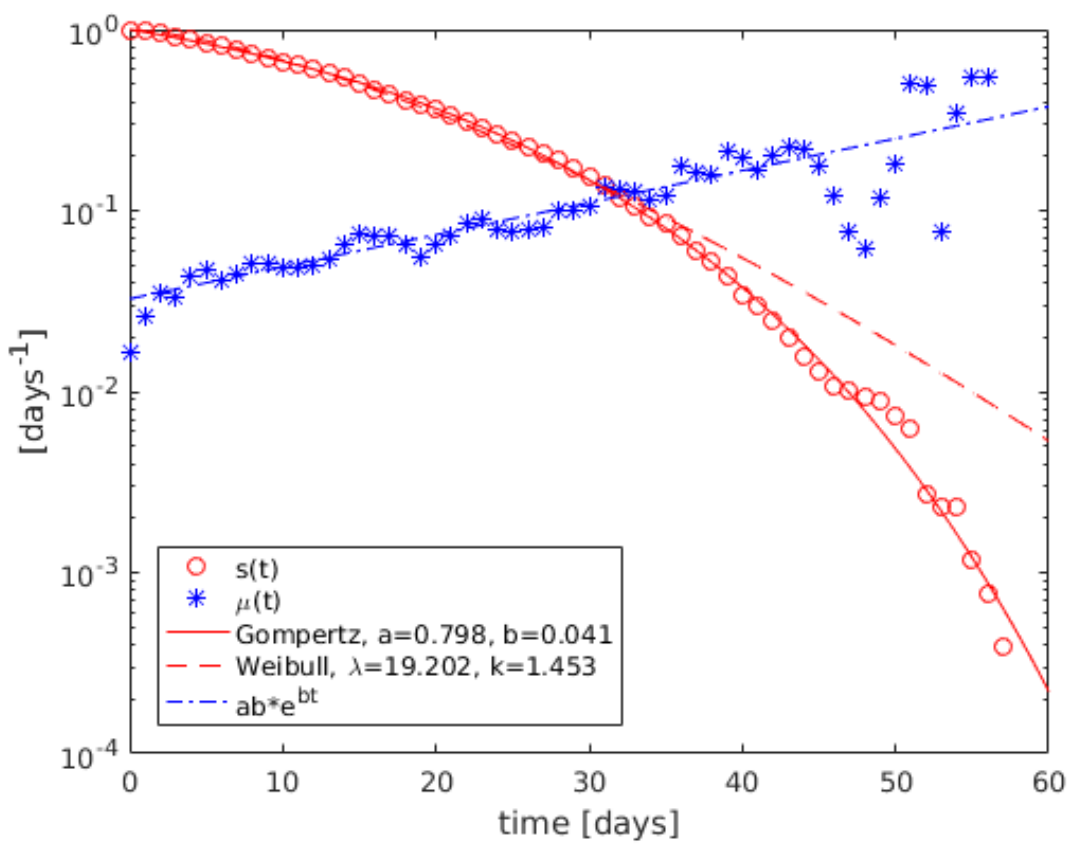

Figure 2. Experimental data for life-time of 2586 soap films contained in 150 tubes, sealed at both ends (Tobin et al. 2011). (a) A fit of the survival function $s(t)$ to a Gompertz distribution (Eqn(5)) is superior to the fit to a Weibull distribution (Eqn(6)) that was proposed by Tobin et al. Note that both functions have only two fit parameters. Also the corresponding mortality rate $\mu(t)$ shows that the data adheres to the Gompertz law for nearly the entire time range.

The fact that collections of soap films confined in tubes may be associated with the Gompertz law, led us to conduct more experiments, under modified experimental conditions, as detailed below, and with more focus on the initial stages of the experiment. This new data set is presented in Figure 3; it reproduces both the bath-tub shape and the exponential increase in mortality/failure rate at longer times, as observed in human mortality data. As we show later, the data also seems to exhibit in the early region the same 
parabolic or power law behaviour characteristic of humans. So it really does seem as if soap films do form a suitable experimental physical system to study the statistics associated with mortality.

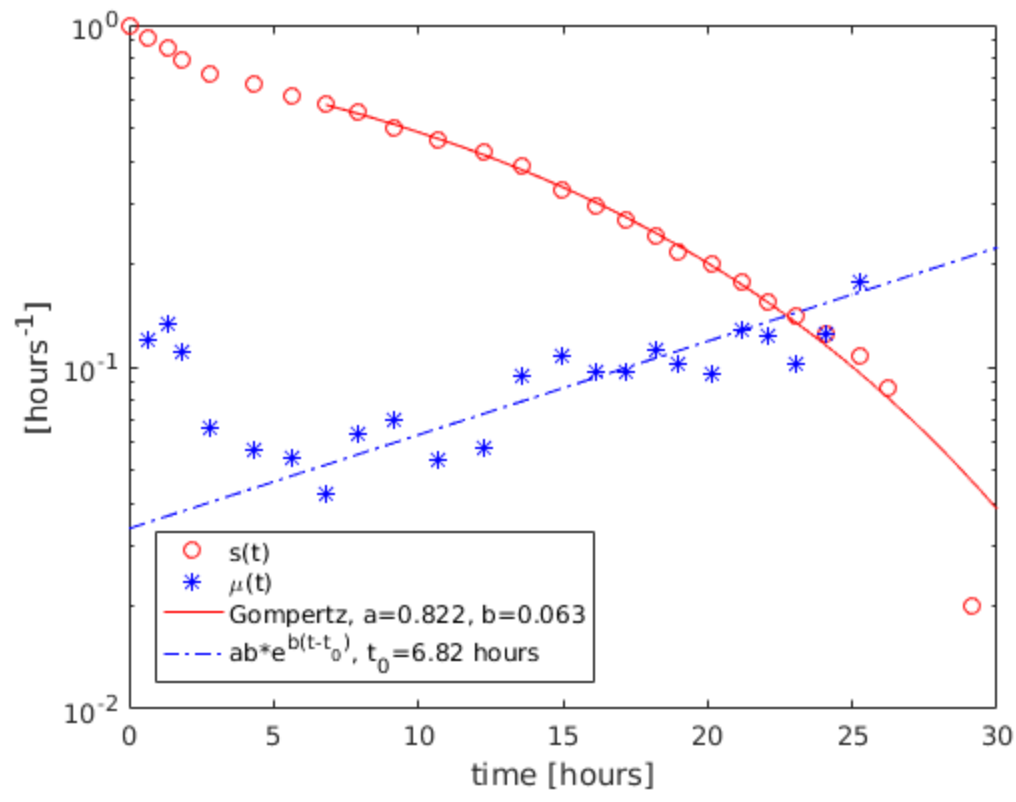

Figure 3. Variation of survival function $s(t)$ and mortality rate $\mu(t)$ obtained from experimental data for 2260 soap films contained in 143 vertical tubes which were sealed at only the bottom end. The mortality rate features a bath tub shape, as is familiar from the variation of human mortality in the range from infant to teenage years (see figure 1). Data in the range from 7 to 25 hours corresponds to ageing; it is broadly described by a fit to the Gompertz distribution (solid line), Eqn(5), with the corresponding $\mu(t)$ as dashed line.

In the next section we outline the experimental method leading to the data shown in Figure 3. The results are discussed in section 3, and we propose a simple model that reproduces the bath-tub shape of the force of mortality $\mu(t)$ in section 4 , before concluding in section 5 .

\section{Experimental set-up and procedure}

As in our previous study (Tobin et al. 2011), we filled transparent perspex cylinders (diameter $2 \mathrm{~cm}$, length $20 \mathrm{~cm}$ ) with roughly equally spaced films 
(about one centimetre between two consecutive films), produced from an aqueous solution of commercial detergent (Fairy Liquid). The films remain attached to the tube perimeter, forming what is called a 'bamboo structure' (Weaire and Hutzler, 1999), until they rupture. While previously we sealed off both tube ends, in our new set of experiments only one end was closed with a cork stopper; the other end was left open to the atmosphere.

A photograph of the apparatus is shown in Figure 4. In each experimental run thirteen cylinders were filled with soap films by dipping each in term into soap solution and manually creating about 16 films per tube. The tubes were then placed vertically and remained so for the entire experiment.

Note that for our purposes, the detailed chemical nature of the surfactant solution used is immaterial. We only require that the film lifetimes are such that they lie within the capability of our measuring apparatus, so that we can see both short and long time behaviour. Any typical commercial soap solution seems to be suitable. Films produced with a chemically well-defined SDS surfactant solution turned out to be too unstable, making it impossible to fill the perspex tubes with a large enough number of films.

The cylinders were placed on a rack in order that the films could be videoed with a digital camera and, following image processing as described below, counted as a function of time. This experiment was repeated 11 times, so a total of 143 cylinders were filled with films, leading to the monitoring of the rupturing of 2260 films rupturing over time. The size of this data set is comparable to our previous experiment, where a total of 2586 films were monitored (Tobin et al., 2011). 
a)

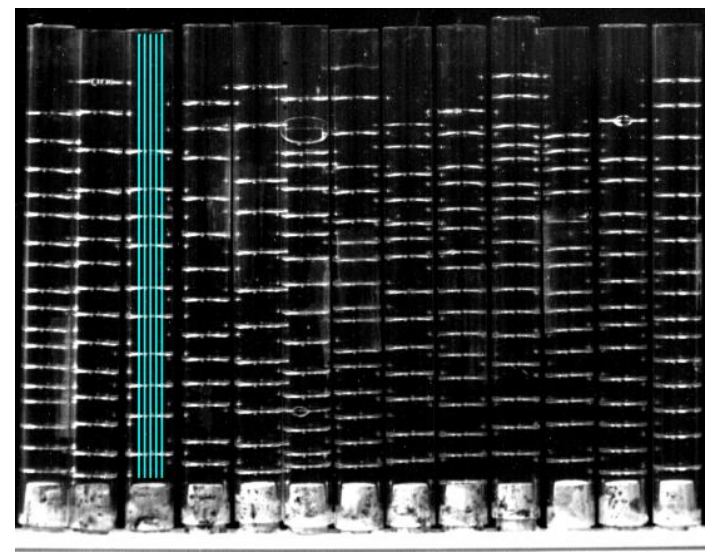

c)

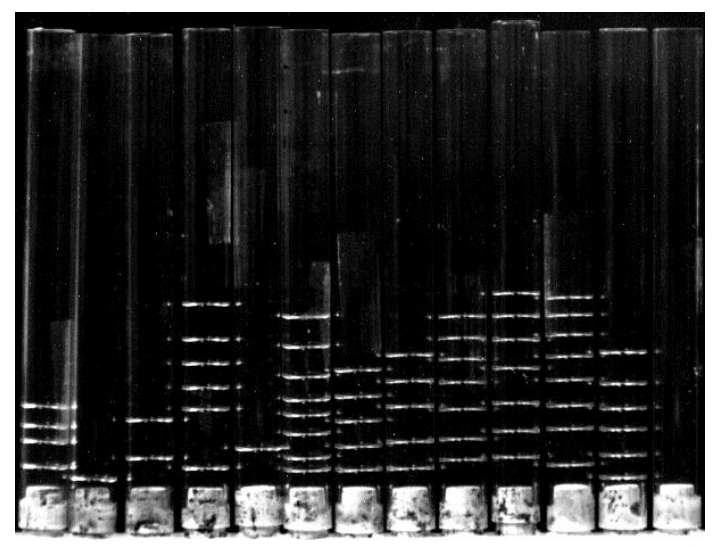

b)

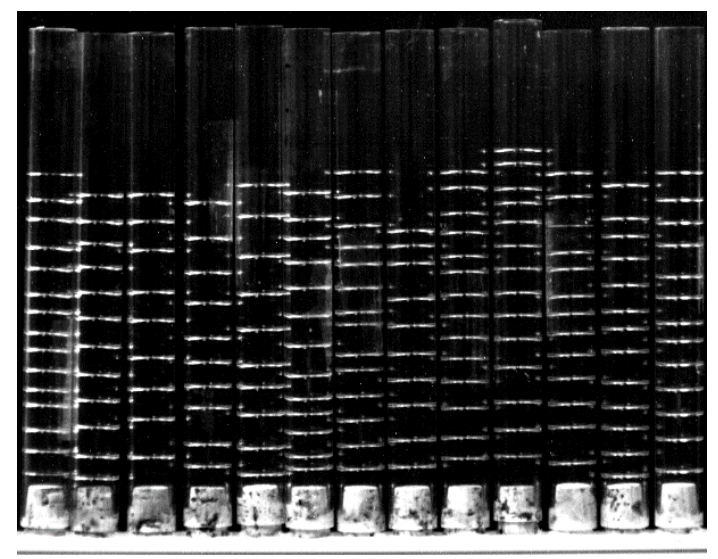

d)

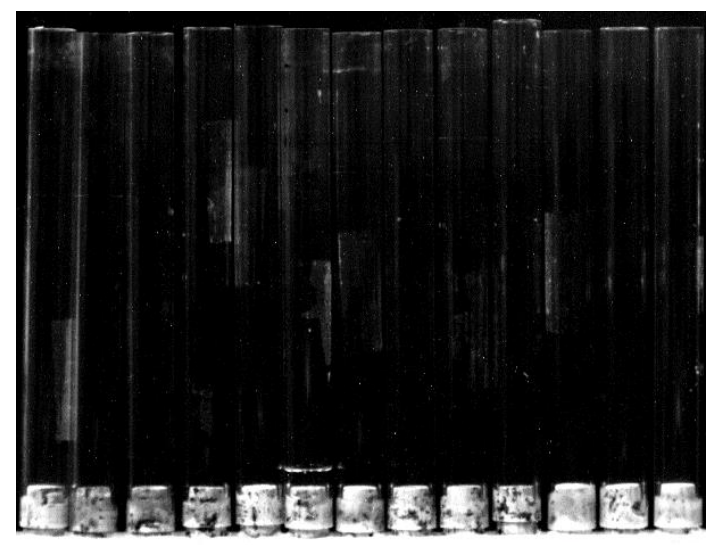

Figure 4. Four still frames extracted from the video at time $t=0(a), t=5(b), t=24$ (c) and $t=31$ hours (d) of one of our 11 experiments. Each of the 13 cylinders is sealed at the bottom with a cork stopper; the top is left open. The cylinders shown contain a total of 194 films initially. Over time both presence and position of the films are followed using image analysis. This is carried out by monitoring the grey values of 5 lines running in parallel to the tubes (as indicated in image (a)). The images show that film rupture propagates mainly from the open end at the top, towards the closed end at the bottom. This indicates that rupturing is driven mainly by liquid evaporating from the film closest to the open top. Image (d) shows the final remaining film in this experiment after 31 hours, just one minute before it too ruptures.

Figure 4 shows four snapshots of an experimental run. The digital images were analysed using a MATLAB script which deduced the vertical position of each film from the distribution of grey values of the images, taken and 
averaged along lines parallel to the tube axis. The lines were each one pixel wide, and the data was averaged over five lines, as shown in figure 4 (a).

The position in the stack of the video file gave the time associated with the position of each film. In this way we could plot the position of each individual soap film as a function of time, even if they slipped during the experiment (which they usually did not). Film rupture leads to a white film disappearing, leaving the black background. In this way we detected both the position of the film that ruptured, and the time of rupture.

We then combined all the data sets from the 11 experimental runs to compute obtain $N(t)$, the number of films of the accumulated runs, as a function of time, where we used a bin size of 50 minutes. The survival function $s(t)$, i.e. the reduced number of remaining films as a function of time, is then obtained via Eq. (1). The mortality function $\mu(t)$, was computed by taking numerical derivatives using the central difference method, see $\operatorname{Eqn}(2)$.

\section{Results and discussion}

Figure 3 shows both survival function and the force of mortality for our combined data set. From the log-linear plot, we see the shape of the force of mortality is very similar to that of mortality data for humans, as shown in the example of figure 1. Unlike the results of our 2011 experiments (figure 2), we now see clear evidence of a 'bathtub' style curve. There is an initial 'infant' region, extending over the first 5-6 hours, where the force of mortality falls. It then reaches a minimum, before it rises again. To a first approximation the rise in this ageing region is linear, as illustrated by the dashed blue line, in agreement with the law of Gompertz. At around 25 hours, the survival data exhibits a steep fall. This suggests we are watching a finite length cut-off which becomes evident as the number of films in the cylinder ultimately 
tends to zero. (The last point shown in Fig 3 represent 45 films.) For longer cylinders which contain many more films we would expect the "linear" region to extend out to longer times before any cut-off.

As with the 2011 data displayed in Figure 2, it is difficult to make any comment on the way the force of mortality behaves very close to the 'end of life'. In this sense both plots mirror human data and more data points are required to draw any conclusions.

Of course the end effect is only relevant to the soap film system. Of greater interest here is the form of the mortality curve in the early stage prior to the force of mortality reaching its minimum point, as shown in figure 3 . Figure 5 shows the data in this region on a log-log plot which appears roughly linear over this region. The same figure shows an exponential decay fit which also describes roughly our data but has no particular relevance to the decay in human populations.

A)

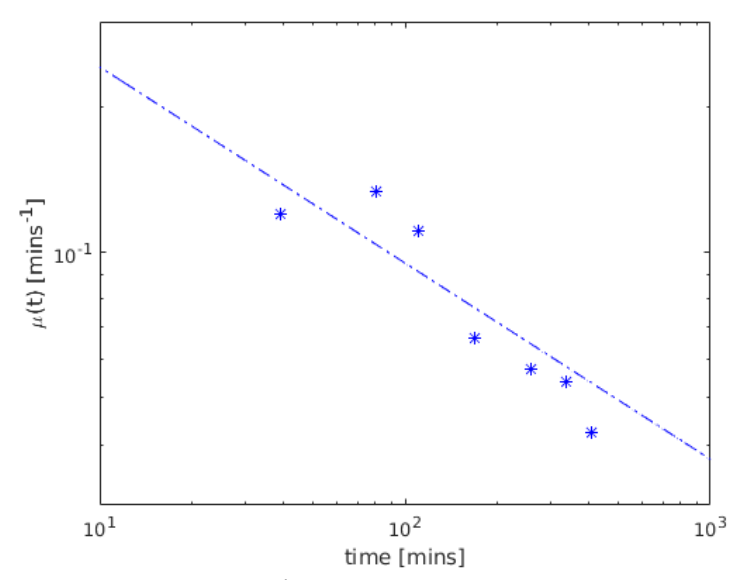

B)

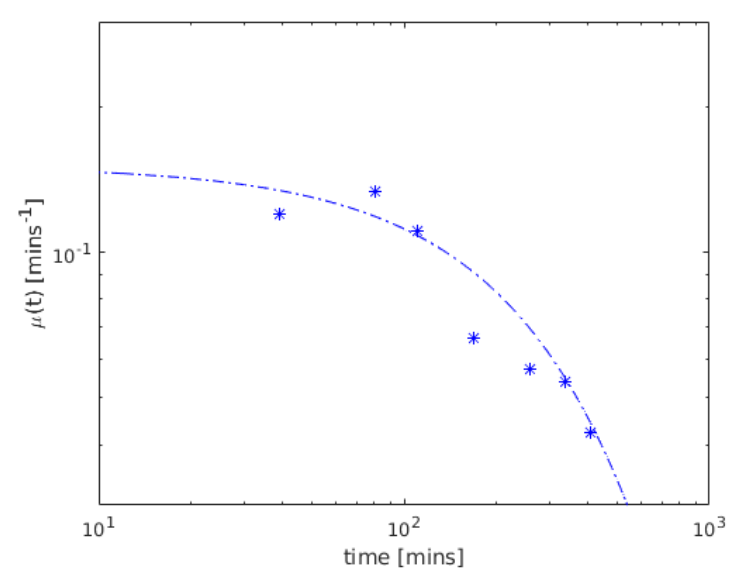

Figure 5. Data for the early stage mortality of the soap film systems shown in figure 3, now plotted on a log-log scale. A) The roughly linear decay is very typical of the behaviour of human systems. B) Exponential decay fit to the data. 
What is the origin of the bath-tub curve in the case of our soap films, contained in the open tube? Comparing the 2011 data for fully sealed tubes (where films lasted up to 2 months) to our new data set, it is obvious that the film life-time is greatly diminished if the tubes are open on one side. The role that evaporation plays was already discussed by Tobin et al. (2011). In their experiment, leakage seemed to occur via the stoppers, leading to sequential rupture from the ends of the tubes towards the centre. However, this was not systematically monitored at the time.

From our new experiments we now have detailed data for the position of all the films and we can compute the film average life-time as a function of its position within the tube, see Fig 6 . Apart from a few exceptions, film rupture does propagate from the open to the closed end of the tube, with a film generally rupturing only once all films above it have also ruptured.

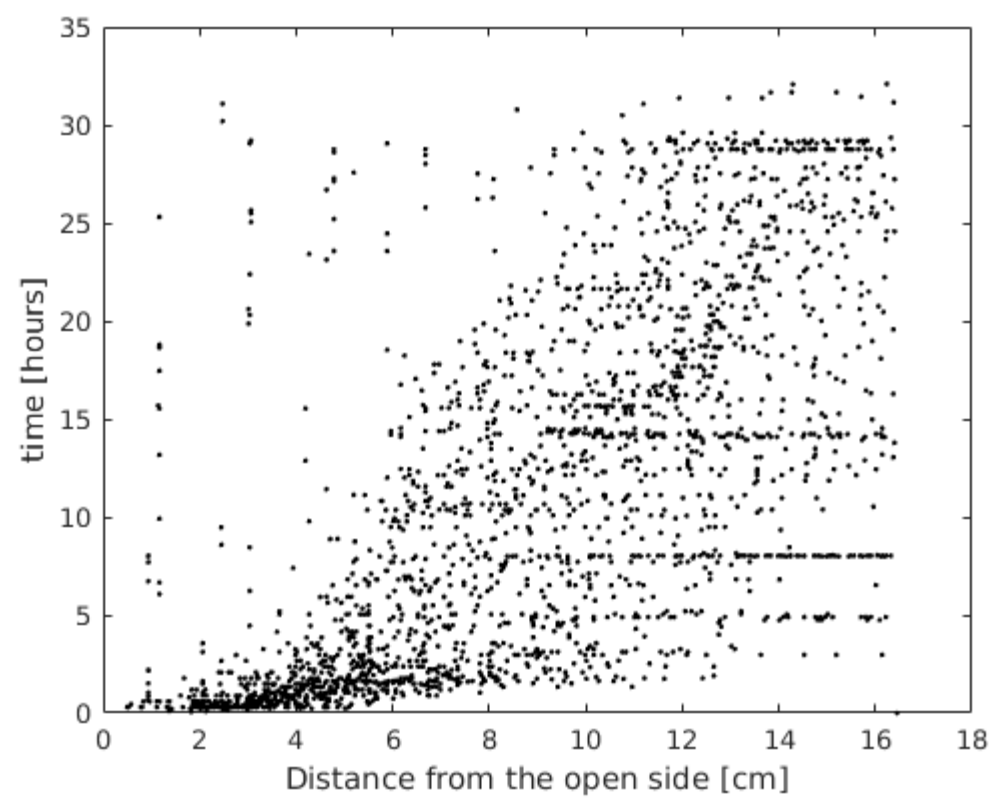

Figure 6: The time for film rupture plotted as a function of film position (distance from the open side) for all the 2260 films contained in a tube. In the first five hours most of the films rupture near the open end of the tube. The rupture time increases roughly quadratically with distance, see also Eqn(7). 
The detailed physical chemistry of soap films is complicated, and there is no universally accepted model for the mechanism of film rupture. However broadly, liquid drainage and evaporation leads to the thinning of films, leaving it prone to rupture as a consequence of mechanical agitation, air currents and thermal fluctuations.

We have investigated this further by studying tubes that contained only one film each, placed at specified locations. The average life-time of films situated at about $1 \mathrm{~cm}$ away from the open end was found to be about 40 minutes (with a standard deviation of 35 minutes), while films situated 16 $\mathrm{cm}$ from the open side lasted on average 7.6 hours (with a standard deviation of 6.6 hours). Figure 7 shows the life-time of films as a function of position for a total of 225 films. The average of film lifetimes increases roughly linear with distance away from the open end of the tube.

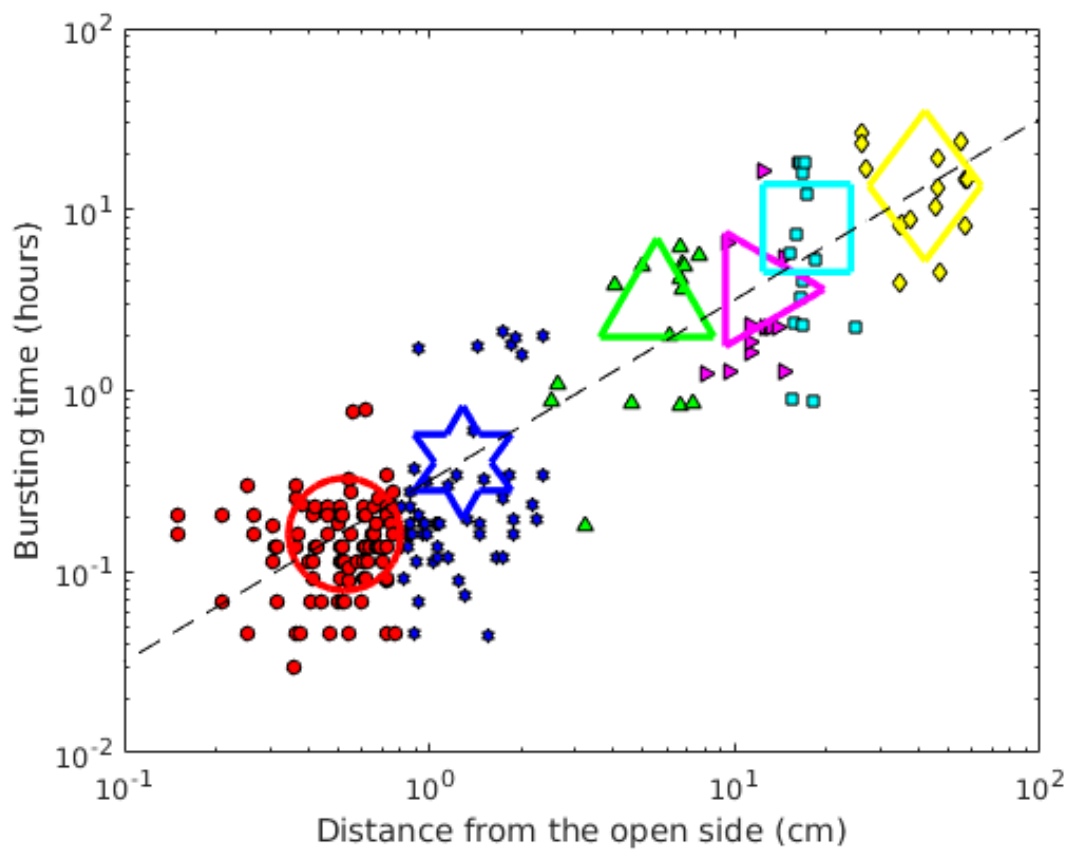

Figure 7. Experimental data for the life-time of single films confined in individual tubes at different positions. The large symbols correspond to data averages for films at similar positions. The dashed line has slope one, indicating that the film lifetime increases roughly linearly with distance away from the open end. 
It is also instructive to look at the lifetime distribution of such films. This requires a sufficiently large data set and we have chosen to look in detail only at 150 films placed $1 \mathrm{~cm}$ away from the open tube since they have the shortest lifetimes. Figure 8 appears to show that in this case, where there is no interaction between films, the Weibull distribution provides a better fit than the Gompertz distribution. There has been speculation that a group of defect free individuals might follow a Weibull mortality distribution but no data exists to support the assertion. The soap film system does allow us to explore this phenomenon in more detail.

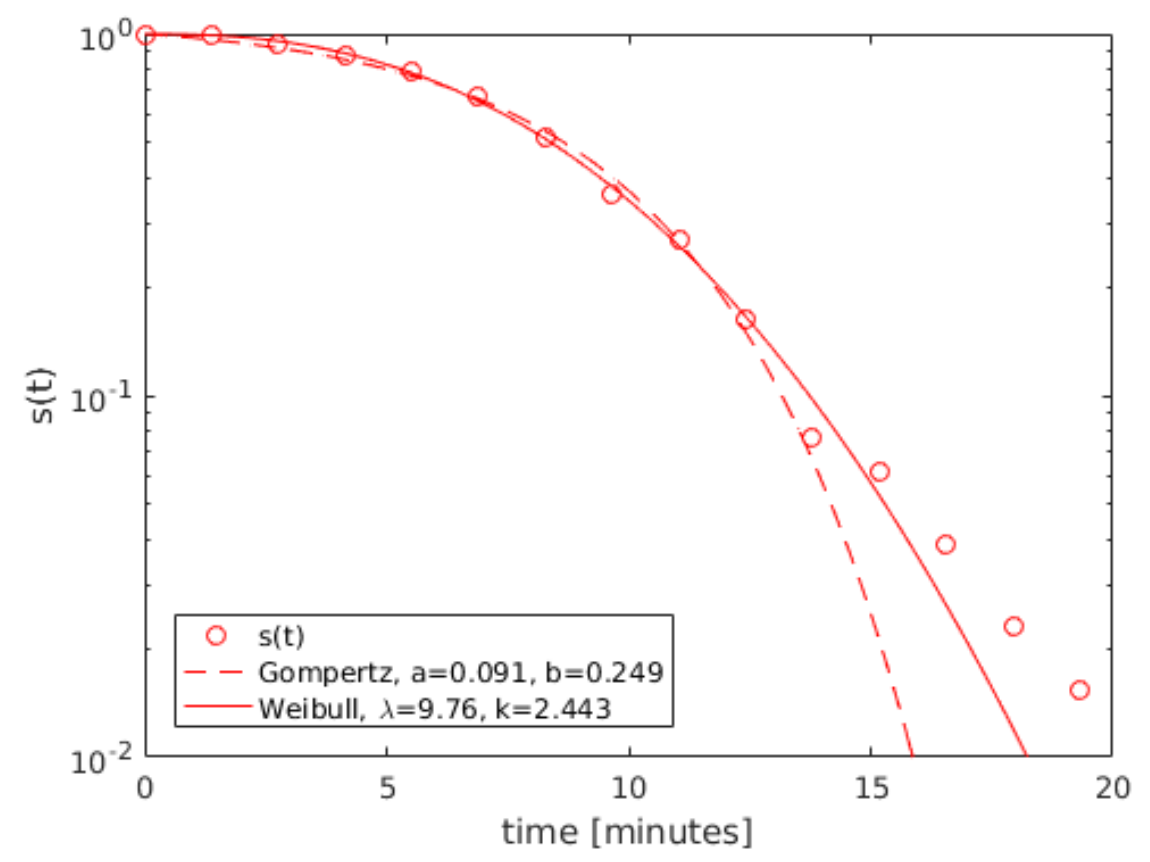

Figure 8. Survival function $s(t)$ constructed from 150 single films in individual tubes, placed about $1 \mathrm{~cm}$ from the open side. This limited data appears to suggest that the Weibull distribution, Eqn(6) (solid line) provides a better fit than the Gompertz distribution Eqn(5) (dashed line). 


\section{A simple model which reproduces the bath-tub shape of the force of mortality}

Let us at this stage return to the most intriguing feature of the force of mortality for our collections of films and ask the question, what is the reason for the bathtub shape of $\mu(t)$ in the initial phase of the experiment? A simple model for this can be constructed following two assumptions, which are supported by the data presented above. These are firstly, that rupture is ultimately due to evaporation of liquid from the thin liquid films, and secondly, that the evaporation rate decreases with the distance away from the open top. This takes into account our experiments with single soap films which showed that their life-time is strongly dependent on their distance away from the open tube end. Finally we demand in our model that a film can only rupture if all films above it have ruptured.

Figure 9 shows a sketch of the geometry, films of equal thickness are placed at equidistant positions $z_{i}=i \Delta h$ inside a cylinder, which is open at the top $(z=0)$ and closed at the bottom. 


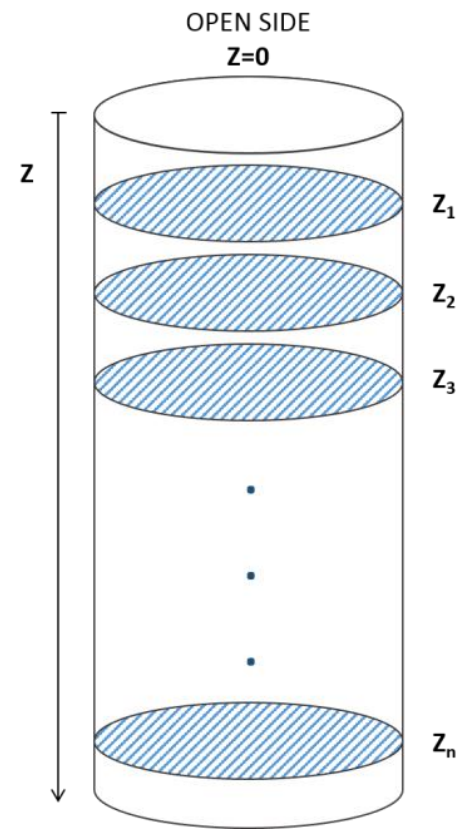

Figure 9. In our simple model films of equal thickness are placed at equidistant position $z_{i}$ perpendicular to the tube axis. The top of the tube at $z=0$ is open, the bottom of the tube is closed. A film at position $z_{n}$ can only rupture if all other films above it have ruptured. The evaporation rate decreases inversely with position $z$.

Away from the open end the evaporation rate of liquid decreases with distance $z$ as $r(z) \sim 1 / z$ as a consequence of Fick's law of diffusion. The life time $T_{1}$ of the first film at $z_{1}$ is thus proportional to $r\left(z_{1}\right)^{-1}$, i.e. $T_{1} \sim r\left(z_{1}\right)^{-1} \sim z_{1}$.

In our simple model the second film will only start evaporating, once the first film has ruptured. The life time $T_{2}$ of the second film, located at $z_{2}$, is thus given by $T_{2} \sim r\left(z_{1}\right)^{-1}+r\left(z_{2}\right)^{-1} \sim \Delta h+2 \Delta h$. By the same argument we obtain for the life-time of the $n$-th film,

$$
T_{n} \propto \Delta h \sum_{i=1}^{n} i=c n(n+1) / 2, \quad \text { Eqn. (7) }
$$

where $c$ is a constant (proportional to the film spacing $\Delta h$ ), with dimension of a time. We note that Eqn.(7) corresponds to a quadratic increase of the 
life-time of a film with its distance from the open side of the tube, consistent with the data shown in figure 6.

The total number of films at time $T_{n}$ is given by $N\left(T_{n}\right)=N_{0}-n$, where $N_{0}$ is the initial number films in the tube. This results in the survival function

$$
s(n)=1-n / N_{0}, \quad \text { Eqn. }(8)
$$

as a function of $n$. To compute $s(t)$ we solve Eqn. (7) for $n$ and insert this into (8). This immediately results in

$$
s(t)=1-\frac{1}{2 N_{0}}(\sqrt{1+4 t / c}-1) \text {. Eqn.(9) }
$$

From this, $\mu(t)$ is readily computed as

$$
\mu(t)=\frac{1}{c}\left[\sqrt{1+4 t / c}\left(N_{0}+1 / 2\right)-\frac{1}{2}(1+4 t / c)\right]^{-1} \quad \text { Eqn.(10) }
$$

Figure 10 shows both $s(t)$ and $\mu(t)$. While this simple model does not give the observed Gompertz law for long times, it nevertheless results in the initial bath-tub shape for $\mu(t)$.

Since we are at this stage only interested in obtaining a general understanding of (soap film) mortality, we have deliberately left out physical constants in the above derivation. These are contained in the constant $c$ and would include diffusivity for a thin liquid film containing surfactants at the interface, film thickness (as a function of time), and humidity; none of these quantities, which all affect film stability (Pugh, 2016), were determined in our experiments. 


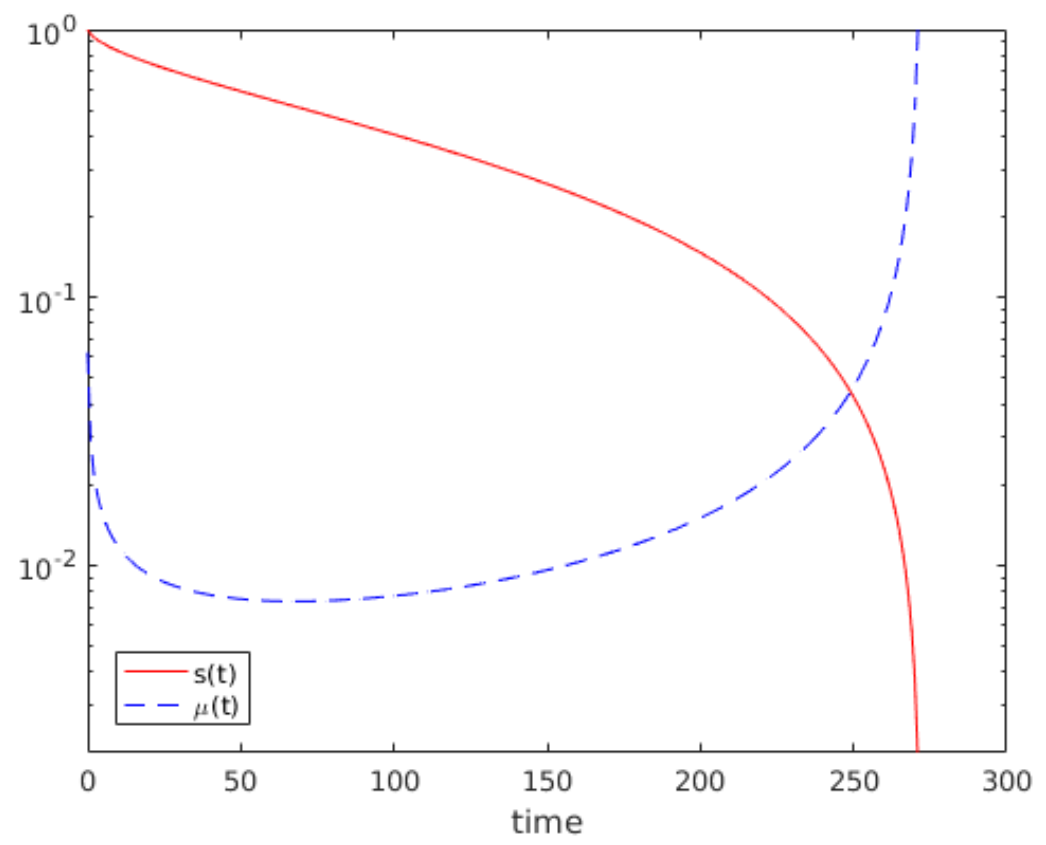

Figure 10: Results of a simple model for lifetime of soap films in tubes, sealed only at one end. $s(t)$ and $\mu(t)$ are plotted using Eq. (9) and Eq. (10) respectively. The mortality function shows a high rate at the very beginning as observed experimentally. (Here $N_{0}=16$ and $\left.c=1 s^{-1}\right)$.

Two predictions, which we intend to probe in future work, follow readily from our model, Eqn.(10). Firstly, the minimum mortality rate is reached at time $t_{m} \approx c N_{0}^{2} / 4$, i.e. it scales linearly with film spacing $\Delta h$, since $c \propto \Delta h$ (see above), and quadratically with the initial film number $N_{0}$ per tube. Secondly, the ratio of the mortality rate at the beginning, $\mu(0)$, and at time $t_{m}$ is given by $\mu(0) / \mu\left(t_{m}\right) \approx N_{0} / 2$. In order to obtain a more drastic decrease in the initial mortality rate this suggests using more films per tube. The time over which this decrease occurs can be stretched further out by increasing the spacing of the films, requiring longer tubes.

Bebbington et al. (2007) develop failure distributions that describe human mortality over the entire time range and apply this to Canadian and Indonesian data. While our model does not describe the long range of our mortality data, it is based on the specific underlying physical mechanisms inherent in our experiments. We hope to evaluate and extend our model with respect to the approach of Bebbington et al. in future work. 


\section{Conclusions}

So far our search of the literature has not revealed any data for a synthetic physical system, which exhibits clearly the features of mortality shown in human lifetime data. The soap film systems studied here demonstrate clearly that in all respects their mortality mimics that of human systems.

But that apart, it seems here now is a system that offers scope to examine a range of other effects, which are observed in human systems. We know the mortality of human systems responds to changes in loss of loved ones or imprisonment (Richmond and Roehner 2016b,c). Do soap films respond to shocks such as environmental change or changes in configuration in a similar manner? Preliminary data, where we have temporarily removed one stopper from a fully sealed tube, show such a response. Human mortality is also susceptible to abnormalities. Soap film systems offer an interesting way to explore these since they can be introduced in a controlled and systematic manner.

Obtaining high accuracy human mortality data for many countries is challenging. For example, the age at death for humans is often not known with precision. Also, since infant death is now greatly reduced in most developed countries, corresponding data is sparse. In comparison, soap films are easy to produce and monitor, they can be used as a reproducible system for studies of failure. The ultimate aim, however, will be to develop an understanding of the key system ingredients that are required to produce the features of human mortality data.

\section{Acknowledgements}

This publication has emanated from research supported in part by a research grant from Science Foundation Ireland (SFI) (Grant no. 13/IA/1926). 


\section{References}

Bebbington, M., Lai, C.D. and Zitikis, R., 2007. Modeling human mortality using mixtures of bathtub shaped failure distributions. Journal of Theoretical Biology, 245(3), pp.528-538.

Berrut, S., Pouillard, V., Richmond, P. and Roehner, B.M., 2016. Deciphering infant mortality. Physica A: Statistical Mechanics and its Applications, 463, pp.400-426.

Davis, D., 1952. An analysis of some failure data. Journal of the American Statistical Association, 47(258), pp.113-150.

Finch, C.E., 1994. Longevity, senescence, and the genome. University of Chicago Press.

French Institute for Demographic Studies (INED). Mortality related to gender and age in France in 2015.

https://www.ined.fr/en/everything about population/data/france/deaths-causes-mortality/mortality-rates-sex-age/

Gompertz, B., 1825. XXIV. On the nature of the function expressive of the law of human mortality, and on a new mode of determining the value of life contingencies. In a letter to Francis Baily, Esq. FRS \&c. Philosophical transactions of the Royal Society of London, 115, pp.513-583.

Gavrilov, L.A. and Gavrilova, N.S., 2001. The reliability theory of aging and longevity. Journal of Theoretical Biology, 213(4), pp.527-545.

Kirkwood, T.B., 2015. Deciphering death: a commentary on Gompertz (1825) 'On the nature of the function expressive of the law of human mortality, and on a new mode of determining the value of life contingencies'. Phil. Trans. $R$. Soc. B, 370(1666), p.20140379.

Pugh, R.J., 2016. Bubble and foam chemistry. Cambridge University Press.

Richmond, P. and Roehner, B.M., 2016. Predictive implications of Gompertz's law. Physica A: Statistical Mechanics and its Applications, 447, pp.446-454.

Richmond, P. and Roehner, B.M., 2016. Effect of marital status on death 
rates. Part 1: High accuracy exploration of the Farr-Bertillon effect. Physica A: Statistical Mechanics and its Applications, 450, pp.748-767.

Richmond, P. and Roehner, B.M., 2016. Effect of marital status on death rates. Part 2: Transient mortality spikes. Physica A: Statistical Mechanics and its Applications, 450, pp.768-784.

Ruby, J.G., Smith, M. and Buffenstein, R., 2018. Naked mole-rat mortality rates defy Gompertzian laws by not increasing with age. eLife, 7:e31157.

Shklovskii, B.I., 2005. A simple derivation of the Gompertz law for human mortality. Theory in Biosciences, 123(4), pp.431-433.

Stauffer, D., 2004. The complexity of biological ageing. In Novak, M.M. (ed.), 2004. Thinking in Patterns: Fractals and related phenomena in nature. World Scientific (pp. 131-142).

Tobin, S.T., Meagher, A.J., Bulfin, B., Möbius, M. and Hutzler, S., 2011. A public study of the lifetime distribution of soap films. American Journal of Physics, 79(8), pp.819-824.

Weaire, D. and Hutzler, S., 2001. The physics of foams. Oxford University Press.

Weibull, W., 1951. A statistical distribution function of wide applicability. Journal of applied mechanics, 18(3), pp.293-297.

Yang, J. and Sun, F.B., 1999, January. A comprehensive review of hard-disk drive reliability. In Reliability and Maintainability Symposium, 1999. Proceedings. Annual (pp. 403-409). IEEE. 